\title{
Traumatic rupture of the pregnant uterus after motor vehicle accident: A case report
}

\author{
Ahmed Belkouch ${ }^{1,}$, Abdelilah Mouhsine ${ }^{2}$, Rachid Sirbou ${ }^{1}$, Abdlghani EIFikri ${ }^{2}$, Lahcen Belyamani $^{1}$ \\ ${ }^{1}$ Emergency Department, Mohamed V Military Hospital of Instruction, Faculty of Medicine and Pharmacy, Rabat, Morocco \\ ${ }^{2}$ Department of Radiology, Avicenna Military Hospital, Faculty of Medicine and Pharmacy, Marrakech, Morocco
}

\section{Email address:}

belkouch1@gmail.com (A. Belkouch), abdelilahmouhsine@yahoo.fr (A. Mouhsine), belyamani@gmail.com (L. Belyamani)

\section{To cite this article:}

Ahmed Belkouch, Abdelilah Mouhsine, Rachid Sirbou, Abdlghani ElFikri, Lahcen Belyamani. Traumatic Rupture of the Pregnant Uterus after Motor Vehicle Accident: A Case Report. International Journal of Medical Imaging. Vol. 2, No. 6, 2014, pp. 131-132.

doi: 10.11648/j.ijmi.20140206.11

\begin{abstract}
The most common obstetric complications after severe trauma in pregnancy are miscarriage, premature labor and premature rupture of membranes. Uterine rupture is rare and it occurs in less than $1 \%$ of pregnant women victims of this kind of accidents. We describe the case of a 36 years old woman victim of a tragic motor vehicle accident. Shock and unconsciousness were the principal symptoms and uterine rupture was discovered at CT scan. A conservative surgical treatment was attempted. The prognosis remains poor and improving it requires the improvement of pre-hospital care.
\end{abstract}

Keywords: Pregnancy, Motor Vehicle Accident, Severe Trauma, Uterine Rupture

\section{Introduction}

Motor vehicle is the most common cause of severe trauma in pregnancy, which is itself the first cause of non-obstetrical maternal death in this context (1). Physiologic modifications in pregnancy can delay the diagnosis of the uterine rupture and the first sign can be fetal distress. We present a rare case of uterine rupture complicating a motor vehicle trauma. The management must be fast in order to improve the prognosis.

\section{Case Presentation}

A 36-years old woman with no significant history has been admitted to the emergency department after a motor vehicle accident; she was a backseat passenger victim of a violent motorcycle collision with a bus. The motorcycle conductor was found dead.

The patient was admitted unconscious GCS $8 / 15$, the blood pressure was $65 / 32 \mathrm{mmHg}$, heart rate was 160 cycles/min, breathing rate was at 32 cycle/min, the abdomen was soft without guarding, Head, neck, and chest examination were unremarkable. Resuscitation maneuvers were performed (2 venous accesses were taken, 2 liters isotonic saline perfusion, norepinephrine, orotracheal intubation and assisted ventilation, blood transfusion). A fast ultrasound showed an important intra-abdominal effusion located in the mourisson, in the splenorenal space, and in the rectouterine pouch of Douglas.

After the hemodynamic was stabilized, we completed imaging by a total body scan which showed in the peritoneum and just behind the abdominal wall muscles the presence of a heterogeneous fluid density formation containing tissue and bone, grossly ovoid measuring about $12 \mathrm{~cm} / 6 \mathrm{~cm}$ associated with dense and fluid intraperitoneal effusion, estimated to 2 liters. These findings are corresponding to an intraperitoneal embryo associated with haemorrhagic effusion (figure 1).

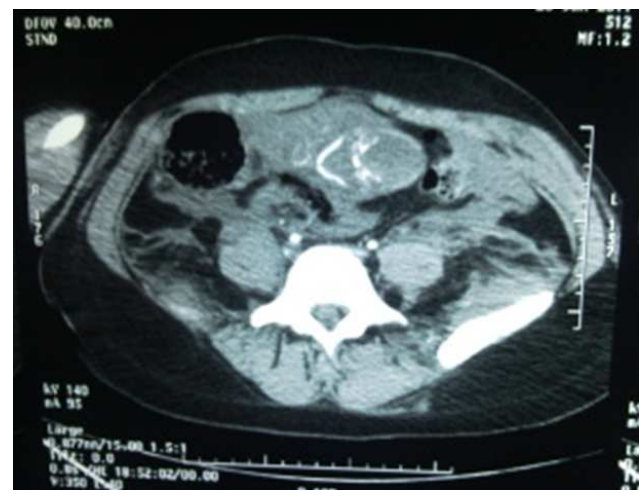

Figure 1. CT scan in axial section after injection of contrast material showing in the peritoneum and just behind the abdominal wall muscles the presence of a heterogeneous fluid density formation within tissue and bone grossly ovoid measuring about $12 \mathrm{~cm} / 6 \mathrm{~cm}$ associated with dense and fluid intraperitoneal effusion. 
The patient was then immediately transferred to the operating room to undergo an exploratory laparotomy which objectified uterine rupture located in the fundus and in the peritoneal cavity the dead fetus and the placenta; no other organs injury was found. A senior gyneco-obstetrician surgeon has then been called and a conservative treatment was attempted.

The patient died two days after in the intensive care unit due to disseminated intravascular coagulopathy; some family visitors confirmed she was 12 weeks pregnant.

\section{Discussion}

Trauma continues to be the leading non obstetrical cause of maternal death (1), it happens in $5 \%$ to $7 \%$ of all pregnancies $(2-3)$ and the most common cause is motor vehicle accidents (4).

Miscarriage, premature labor and premature rupture of membranes are the most common obstetric complications. (5)

Uterine rupture is rare and it occurs in less than $1 \%$ of pregnant women victims of this kind of accidents (6). Factors promoting the uterine rupture are sudden increases in abdominal pressure and deceleration phenomena (5).

Fetal death occurs in $0.03-0.09 \%$ of these cases (7), its primary cause is maternal death followed by placental abruption (8). According to Weiss \& al, placenta abruption was responsible of $42 \%$ of fetal deaths; $11 \%$ were due to maternal schock or death. (4), both were present in our case.

Physiologic modifications during pregnancy include a $45 \%$ increase in maternal blood volume and a 20 to $30 \%$ increase in cardiac output (8). Vasoconstriction of uterine arteries and a decrease in the in the uterine blood flow are the first changes intending to compensate blood loss as in uterine rupture. This means that the first sign of shock will be fetal distress.

The classic symptoms of uterine rupture are severe abdominal pain associated with defense, severe maternal shock, vaginal bleeding and palpated fetus outside the uterus; however, this clinical picture is sometimes not complete as for our patient admitted with hemorrhagic shock and unconsciousness as principal symptoms.

Abdominal coupled to obstetrical ultrasound is the gold standard for the assessment of both abdominal lesions (intraperitoneal effusion) and fetal vitality. As we did not previously know that the patient was pregnant we performed a classic FAST echo which showed the hemoperitoneum but did not help to detect the fetus as our emergency physicians are not trained to obstetrical ultrasound.

The CT scan is better to the assessment of both maternal and fetal lesions, it can also detect other traumatic lesions but the realization of this review should not delay care if maternal and / or fetal prognosis is in imminent danger (9).

In our case we performed a total body CT scan as indicated for the management of severe trauma which suspected the diagnosis of uterine rupture by the presence of the fetus in the peritoneal cavity with hemoperitoneum.

Controlling bleeding should be the primary goal. Rescue hysterectomy is sometimes necessary. However, each time a preservative treatment can be performed it must be preferred.

The maternal-fetal prognosis remains poor, as in our case, the control of bleeding was not sufficient to save the patient and she died after disseminated intravascular coagulopathy, which is frequent in this context due to physiological modifications; indeed Pregnancy represents an inner transitional prothrombotic state. The prehospital management and rapid rescucitation in the emergency room is critical to reducing morbidity and maternofetal mortality (9).

\section{Conclusion}

Motor vehicle trauma continues to be the leading cause of severe trauma to pregnant women in our country and around the world. Uterine rupture is a rare complication but remains very serious with a poor prognosis. In our country, improving the prognosis depends on improving the pre-hospital care.

\section{References}

[1] Weinber L, Steele RG, Pugh R, Higgins S, Herbert M, Story D. The pregnant trauma patient. Anaesth Intensive Care 2005;33:167-80

[2] Hill CC, Pickinpaugh J. Trauma and surgical emergencies in the obstetric patient. Surg Clin North Am 2008;88:421- 40.

[3] Green W, Robinson L, Rizzo AG, et al. Pregnancy is not a sufficient indicator for trauma team activation. J Trauma 2007;63:550-5.

[4] Weiss HB, Songer TJ, Fabio A. Fetal deaths related to maternal injury. JAMA 2001;286:1863-8

[5] Towery R, English TP, Wisner D. Evaluation of pregnant women after blunt injury. J Trauma 1993;35:731-5

[6] Rowe TF, Lafayette S, Cox S. An unusual fetal complication of traumatic uterine rupture. J Emerg Med 1996;14:173-6

[7] Warner MW, Salfinger SG, Rao S, Magann EF, Hall JC. Management of trauma during pregnancy. ANZ J Surg 2004;74:125-8

[8] Pearlman MD, Tintinalli JE, Lorenz RP. Blunt trauma during pregnancy. N Engl J Med 1990;323:1609-13.

[9] C. Vayssea,b, F. Mignota, J.-P. Benezecha, O. Parantb,*. Rupture utérine traumatique : une complication rare des accidents de la voie publique au cours de la grossesse. À propos d'un cas. Journal de Gynécologie Obstétrique et Biologie de la Reproduction 36 (2007) 611-614 